\title{
Kinetics of Flexibly Fixed Operating Element of a Rotating Device
}

\author{
Zdzisław Pluta, Tadeusz Hryniewicz* \\ Koszalin University of Technology, Raclawicka 15-17, PL 75-620 Koszalin, Poland \\ *E-mail address: Tadeusz.Hryniewicz@tu.koszalin.pl
}

\begin{abstract}
The work covers the adequate kinetics of the flexibly fixed working element of a rotating device. First the literature background of the problem has been presented, turning the attention on such systems where the phenomenon of centrifugal displacement of a body due to the rotating of the system occurs. Then the general characteristics of the complex motion of working element has been described. The core of the work is the description of the variable component motions, with the starting point of the description being the source differential equation, presenting this type of dependence of the path length on time. The accelerated angular motion and retarded radial motion have been separated, describing these component motions as the function of time. Based on these detailed descriptions, the trajectory equation of working element, the derivatives and further kinetic magnitudes, have been derived. In the conclusion, the cognitive and practical qualities of the presented solutions of the title problem, have been accented.
\end{abstract}

Keywords: Kinetics; Susceptibility; Device; Potential field; Time constant; Body trajectory; Path length; Velocity; Acceleration

\section{INTRODUCTION}

The flexible characteristics of fixations of material bodies are multiple and variable, and in fact all they have such nature; thus they may be differentiated and considered from the point of view of scale, and magnitude of the flexibility. Such fixed bodies displace, changing their positions due to external reactions.

This work directs the attention on the displacements of the operating elements of a rotating device, clearly indicating the kinetics of that element. Its motion has a character respectively complex, and that takes place specifically during the start-up of a device comprising such a susceptible system.

It is referred to devices, showing with this essentially broad application. Kinetics of the susceptibly fixed operating element is noticed during the motion of different types of devices, for instance: the elastic grinding wheel, centrifugal start-up coupling, flexible shaft, heads to centrifugal burnishing.

It is worthy generalizing this kinetic quest by analysing it in much more detail; it is quite essential for the further developments of the subject, especially of the dynamics of a flexibly fixed operating element. It is mainly on the consideration and exact description of the 
inertia phenomenon; and that will be possible if the entire, complete kinetics will be taken into account.

In the framework of introduction it is worthy adding the explanation, what is covered under the notion of kinetics. The kinetics covers such a mechanical characteristics of the body, where the body mass is not considered, treating it as a material point only. Kinetics covers then such magnitudes, like: the path length, velocity, acceleration; with all of them characterizing each motion of the body, so variable, and uniform.

\section{LITERATURE BACKGROUND OF THE PROBLEM}

It is worthy now, before entering any subject problem, presenting the literature background; the background showing the system, where the phenomenon of centrifugal body displacement appears due to rotating of the system under a fixation.

Such operating elements may be the abrasive grains, situated on the peripheral surface of an elastic grinding wheel. That special porous abrasive tool, is manufactured by numerous renown companies [1-6]; these grinding wheels have polyurethane bond so the fixed grains in them may displace both during starting the rotating motion as well as during the material machining.

The flexibility of grain fixations on the working surface of elastic grinding wheel is much greater that the flexibility of their fixation in the ceramic grinding wheels. If the displacements of grains in the machining zone during grinding equal over ten micrometers [7] and may be comparable with the cutting depth, so in case of elastic grinding wheels these displacements are considerably bigger and may reach the values even up to one millimeter [8$10]$.

Turning such an elastic grinding wheel into rotating motion results in its radial deformation on the order of some millimeters [10]; however, its special fixing [11], providing more unconstrained deformation in the radial direction makes the radial expansion of the grinding wheel is noticed even on the length of over ten millimeters [12].

The operating element of the rotating tool may be also respectively elastically fixed the grinding wheel segment. That segment in turn may possess a rigid structure, resulting from the characteristics of the ceramic bond; or elastic, caused by the nature of polyurethane bond. The patent description [13] presents the solution of such fixation of the abrasive working element in a special head to the mechanical smoothing treatment.

The example of a practical design solution should be added to the literature background. They are the start-up couplings, clutches, where the flexibly fixed weights/swings which cause coupling, during the rotating motion, of the shaft of electric engine with the driven shaft [14-15].

The essence of this problem may be referred to other technical/technological systems. The examples of a rod material during accelerating rotating motion are also interesting. The material of such a shape would be dynamically extended and the characteristics obtained would have a great significance from the viewpoint of a material strength [16]. Based on the kinetics of such formed material all forces occurring in this rotating rod could be determined. 


\section{GENERAL CHARACTERISTICS OF THE COMPLEX MOTION OF WORKING ELEMENT}

A working element is fixed elastically, represented by a spring, and is in the point 0 , at the distance $r_{0}$ from the center of a device rotation, containing that mass-spring system (Fig. $1)$.

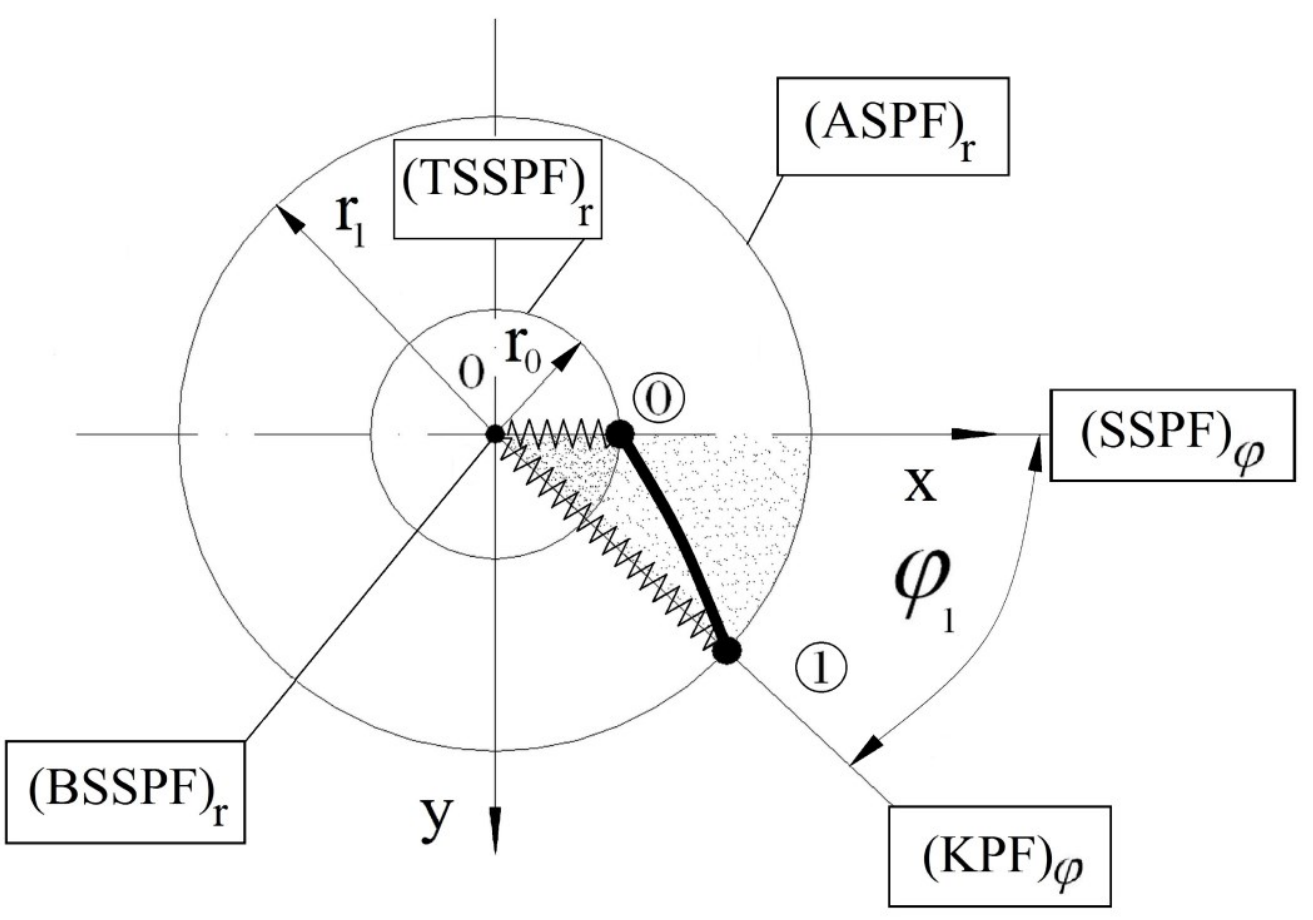

Fig. 1. General characteristics of the complex motion of working element

After the device setting in motion that element begins the motion in radial direction, and displaces, carried by the device case, in angular direction. In both the directions the variable motion takes place; however, in radial direction it has a retarded character (due to retarding action of spring element), and accelerated in the angular direction.

After some time that element will displace angularly of the angle $\varphi_{1}$, and in radial direction of the radius increment $(\Delta r)_{0-1}=r_{1}-r_{0}$, assuming the position corresponding with the point 1 . From the energetic viewpoint one may say the body changed its energetic state, coming through the space-time (dotted area) from one notch of the potential fields to another notch.

That notch is formed of the stable static potential fields: upper radial stable static potential field $(T S S P F)_{r}$ and the angle stable static potential field $(S S P F)_{\varphi}$. Bottom radial stable static potential field $(B S S P F)_{r}$ is concentrated in the rotation centre of the entire device, and between bottom and upper field of this type there is the energetic band (thick dotted area). The second notch is formed of the following fields: radial unstable static potential field $(A S P F)_{r}$ and the angular kinematic potential field $(K P F)_{\varphi}$. This former field is present at the moment of entrance of the considered body on the unstable static orbit. That 
field moves with a uniform motion, so relating to the kinematics, with the name coming out of this field.

It is worthy adding that the notion of the potential fields, understood as the sites of energetic states of the material body, was introduced by the author in the monograph [10]. Furthermore, this notion found its broader application, for instance in [17], where the adequate description of the tool life under cut versus main velocity was done. That dependence, surely the quantum one, was usually approximated by one or two straight lines.

At this stage of considerations one should state that the body trajectory on the path between the neighbouring orbits is non-linear; that results from the composition of two quite different variable motions. Furthermore this trajectory will be exactly described. Now the description of these component motions will be presented.

\section{DESCRIPTION OF VARIABLE COMPONENT MOTIONS}

The starting point to the description of these component motions is the source, general differential equation, covering the record of all variable phenomena of nature; it is contained in the monograph [10], covering the foundations of surface smoothing by means of elastic abrasive wheels. One may add the use of this equation has been presented in the work [17].

The mentioned general differential equation is as follows:

$$
d Z= \pm \frac{\partial Z}{\partial N} d N
$$

where: $d Z$ - total differential of the dependent variable; $d N$ - total differential of the independent variable; $\frac{\partial Z}{\partial N}$ - partial derivative of the dependent variable, referred to independent variable. The signs $( \pm)$ are the algebraic operators, fulfilling a determined role. The sign (+) has a formal meaning, because it just confirms the physical sense of a determined dependence. The sign $(-)$ ascribes such a sense to a determined record.

For the considered variables the formula (1) has the following two forms:

$$
\begin{aligned}
& d \varphi=\frac{\partial \varphi}{\partial t} d t \\
& d r=-\frac{\partial r}{d t} d t
\end{aligned}
$$

because the dependent variables are: the angular path length $\varphi$ and the radial path length $r$, with time $t$ being the dependent variable. As can be seen, the equation of the angular path length has the sign $(+)$, as it touches the accelerated motion. The equation of the radial path length possesses the sign $(-)$; and that means it is referred to a retarded motion.

Now the integrations on both sides of the dependences are to be performed; one should remember the total differential is the state function. It requires now the determination of states 
for both these motions separately. That means the integration limits referred to these states should be defined.

The scheme of creation of the adequate description of the dependence $\varphi=f(t)$ for accelerated motion is presented in Fig. 2, showing all elements of this reasoning process. The curve illustrating that dependence comes out of an initial point of the coordinates $t=0$, $\varphi=0$, and then has exponential and progressively rising course. It completes its non-linear course in the point 1 , where the accelerated body motion has its end; then the linear course takes place, as the motion of the considered element is uniform.

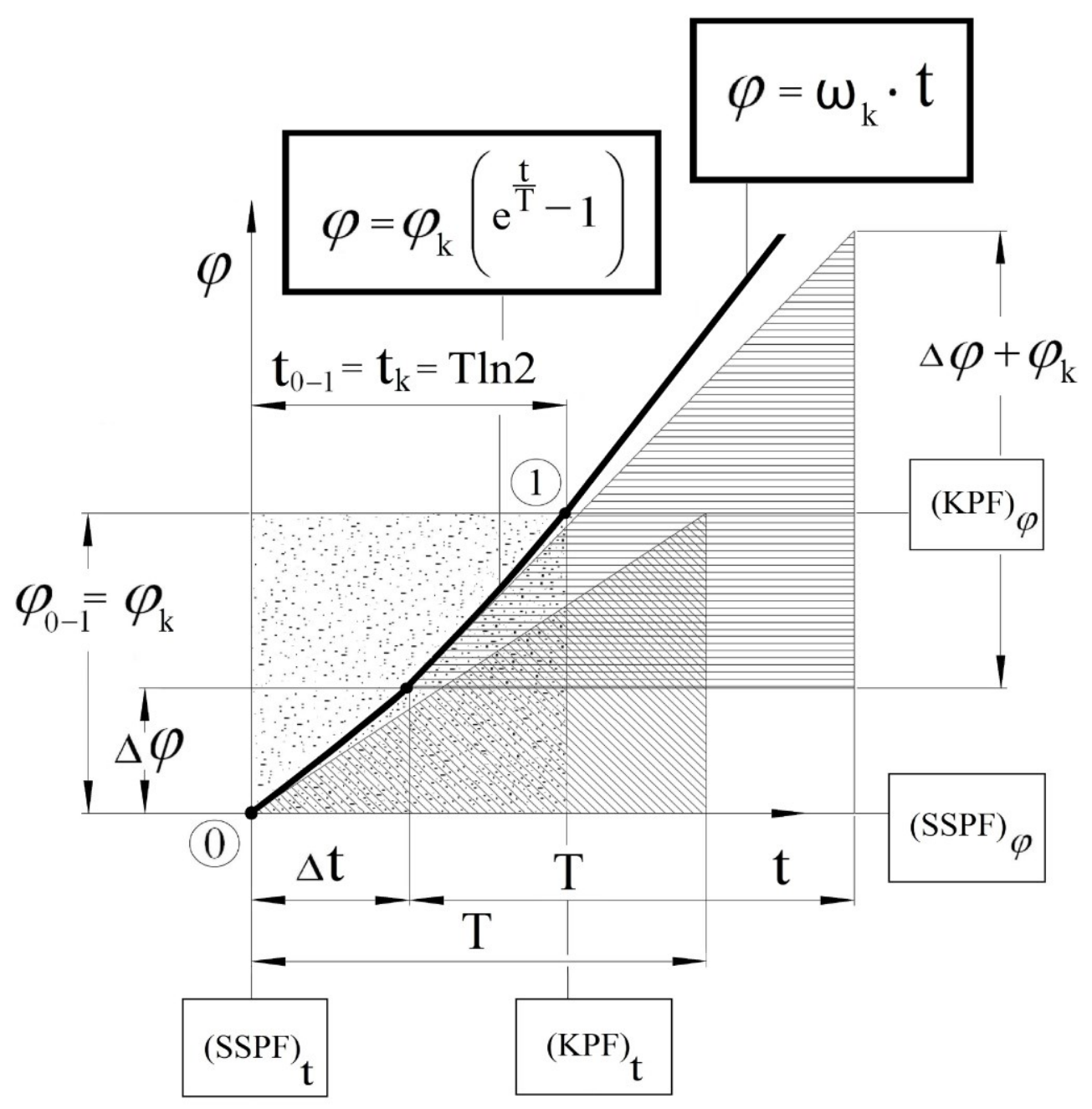

Fig. 2. Angle way characteristics of the working element in its accelerated motion

The mentioned limits, concerning the studied system, are the potential fields. The timespace is limited by these fields both on time and path directions. On the time direction they are the following fields of this type: time stable static potential field $(S S P F)_{t}$, and the time kinematic potential field $(K P F)_{t}$. On the path direction there are the following potential fields: angular stable static potential field $(S S P F)_{\varphi}$, and the angular kinematic potential field 
$(K P F)_{\varphi}$. (It is worthy noticing that the time factor of the considered time-space has been revealed.)

The path length of this time-space $\varphi_{0-1}$, that is the distance between the angular potential fields, is equal to the kinematic path coordinate, that is $\varphi_{0-1}=\varphi_{k}$. The time length $t_{0-1}$ corresponds with the kinematic time coordinate $t_{k}$, so $t_{0-1}=t_{k}$. That last coordinate will be determined closer in the next section.

The curve of the path length, comprised between the points $0-1$, is the envelope of the right-angled triangles, with the time leg being constant and equal the so called time constant $T$, whereas the way leg varies respectively, that results from the changing position of tangent to this curve. For $\Delta \varphi$ the length of this leg equals $\Delta \varphi+\varphi_{k}$.

Now one may come to the integration of the equation (2), marking the limits of the integrals of total differentials. That means

$$
\int_{\Delta \varphi}^{2 \Delta \varphi+\varphi_{k}} d \varphi=\frac{\partial \varphi}{\partial t} \int_{\Delta t}^{\Delta t+T} d t
$$

and further

$$
\Delta \varphi+\varphi_{k}=\frac{d \varphi}{d t} T
$$

or

$$
\frac{d \varphi}{\Delta \varphi+\varphi_{k}}=\frac{1}{T} d t
$$

One may notice, the partial derivative has been substituted by the quotient of the total differentials. It could be done that way because the total differentials have been clearly determined through the introduction of limits of the integrals.

Furthermore, by integrating both sides of the equation (6), one obtains the following result

$$
\ln \left(\Delta \varphi+\varphi_{k}\right)=\frac{1}{T} t+C^{*}
$$

that is

$$
\Delta \varphi+\varphi_{k}=e^{\frac{t}{T}+C^{*}}=e^{C^{*}} \cdot e^{\frac{t}{T}}=C e^{\frac{t}{T}}
$$


After considering that for $t=0$ the magnitude $\Delta \varphi=0$, one obtains

$$
C=\varphi_{k}
$$

and after substituting (9) to (8)

$$
\Delta \varphi=\varphi_{k}\left(e^{\frac{t}{T}}-1\right)
$$

or simply

$$
\varphi=\varphi_{k}\left(e^{\frac{t}{T}}-1\right)
$$

as in the initial point $\varphi_{0}=0$, then

$$
\Delta \varphi=\varphi-\varphi_{0}=\varphi-0=\varphi
$$

Now one may determine the second coordinate of the point 1 , that is the time length $t_{k}$. That result is obtained through the introduction the path length $\varphi=\varphi_{k}$ and the mentioned time length $t_{k}$ to the equation (11). Therefore

$$
t_{k}=T \ln 2
$$

The angular path length out of this time-space, that is in the uniform motion, is expressed by the following dependence:

$$
\varphi=\omega_{k} \cdot t
$$

where the symbol $\omega_{k}$ corresponds with the kinematic angular rate.

It is time now to present the scheme of creation of the adequate description of the dependence $\varphi=f(t)$ for a retarded motion (Fig. 3), so in the radial direction. The curve illustrating that dependence comes out of the initial point of the coordinates $t=0, r=r_{0}$, and further it has exponential and degressively rising course. It completes its non-linear and real/physical course in the point 1 , where the retarded motion of the considered element has its end; further that real course is invariable, and constant. One may notice further, non-real, 
fictional, just mathematic fragment of the non-linear course (dashed line), has been introduced. The need of introduction of such further fragment of the curve will be clarified in the next section.

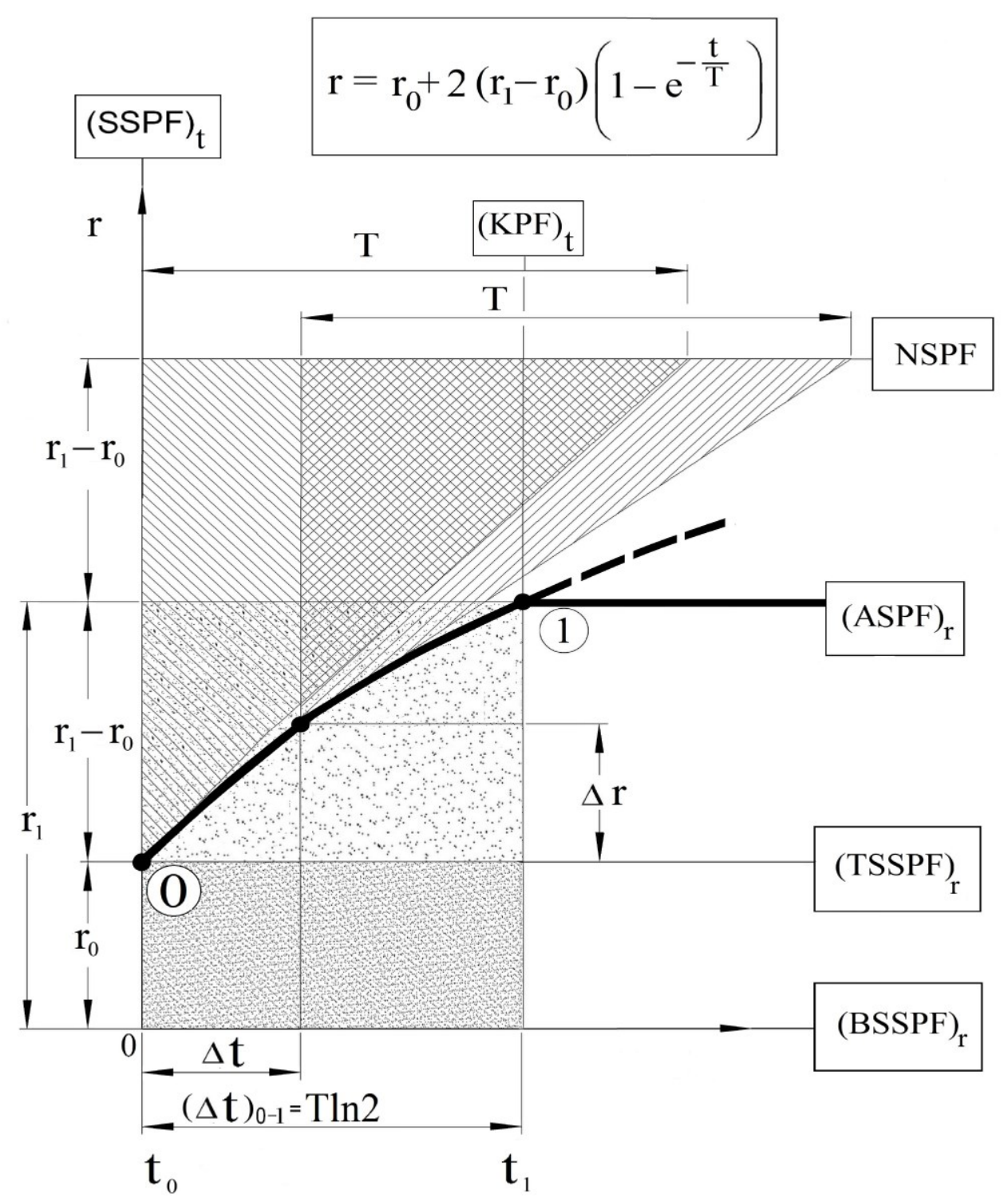

Fig. 3. Radial path characteristics of the working element in its retarded motion

Now the integration of the dependence (3) on both sides should be performed; with this, one should remember that the total differential is the state function. Furthermore, it requires to determine the states, i.e. the integration limits for this retarded motion.

It is known, the mentioned limits are the potential fields. These fields possess their positions on two directions; one of them is the direction of changes of the radial path length $r$, whereas the second one is the direction of time $t$. On this first direction, the following may be noticed: bottom radial stable static potential field $(B S S P F)_{r}$, upper radial stable static potential field $(T S S P F)_{r}$, radial unstable static potential field $(A S P P)_{r}$, and the nominal static potential field $N S P F$. On the second direction, there are the following fields: time stable static 
potential field $(S S P F)_{t}$, time kinematic potential field $(K P F)_{t}$. Between the fields $(B S S P F)_{r}$ and $(T S S P F)_{r}$ the potential band takes place (thick dotted area). The fields $(T S S P F)_{r}$, $(A S P F)_{r}$, and $(S S P F)_{t}$ and $(K P F)_{t}$ limit the proper time-space (loose-dotted area), where the real exponential and degressively rising changes of the radial path length occur. Between the fields $(A S P F)_{r}$ and $N S P F$ there is an improper time-space, fulfilling the auxiliary role (that is an auxiliary mathematic tool).

The curve of radial path length, comprised between the points $0-1$, is the envelope of the right-angled triangle, moving with its horizontal leg on the nominal static potential field $N S P F$; with the horizontal leg being invariable and equal to the time constant $T$, whereas the vertical leg varies respectively, decreasing during displacement of triangle in the time direction. That nominal field is situated symmetrically against the level of the end of the retarded body motion, on the distance equal to the real space-time length in the direction of the radial path length.

Thus beyond the real/proper space the above mentioned improper space is situated, where the curve (dashed line) approaches the asymptote, being this nominal potential field. This creature is an auxiliary design, needed to describe the real curve, reflecting the adequate dependence of the radial path length on time.

Now one may begin the integration of the equation (3). By integrating this equation one should mark the limits of integrals of the total differentials. That means

$$
\int_{r_{0}+\Delta r}^{r_{1}+\left(r_{1}-r_{0}\right)} d r=-\frac{\partial r}{\partial t} \int_{\Delta t}^{\Delta t+T} d t
$$

and further

$$
2\left(r_{1}-r_{0}\right)-\Delta r=-\frac{d r}{d t} T
$$

or

$$
\frac{d r}{2\left(r_{1}-r_{0}\right)-\Delta r}=-\frac{1}{T} d t
$$

One may notice, here the partial derivative has been substituted by the quotient of total differentials. It could be done that way because the total differentials have been clearly determined through the introduction of limits of their integrals.

Furthermore, through the integration of both sides of the equation (17), one obtains the following result

$$
\ln \left[2\left(r_{1}-r_{0}\right)-\Delta r\right]=-\frac{1}{T} t+C^{*}
$$


that is

$$
2\left(r_{1}-r_{0}\right)-\Delta r=e^{-\frac{t}{T}+C^{*}}=e^{C^{*}} \cdot e^{-\frac{t}{T}}=C e^{-\frac{t}{T}}
$$

Regarding that for $t=0$, the magnitude $\Delta r=0$, one obtains

$$
C=2\left(r_{1}-r_{0}\right)
$$

and after substituting (20) to (19), and further, regarding that $\Delta r=r_{1}-r_{0}$

$$
r=r_{0}+2\left(r_{1}-r_{0}\right)\left(1-e^{-\frac{t}{T}}\right)
$$

One may now determine the second coordinate of the point 1 , i.e. $(\Delta t)_{0-1}=t_{1}$. It is achieved through the introduction $r=r_{1}$ and $t=t_{1}$ to the equation (21). Therefore

$$
t_{1}=T \ln 2
$$

Now one can determine the body trajectory, that is the dependence $r=f(\varphi)$, and also further, the derivative kinetic magnitudes. That is to be clearly presented in the following section.

\section{TRAJECTORY OF WORKING ELEMENT AND DERIVATIVE AND FURTHER KINETIC MAGNITUDES}

First of all it is worthy formulating the dependence of the radial path length $r$ on the angular path length $\varphi$, that is $r=f(\varphi)$. It is obtained from the equations (11) and (21), by introducing first $e^{\frac{t}{T}}$ from the equation (11), then

$$
e^{\frac{t}{T}}=\frac{\varphi_{k}+\varphi}{\varphi_{k}}
$$

and further substituting (23) to (21), that leads in consequence to the following result 


$$
r=r_{0}+\frac{2\left(r_{1}-r_{0}\right) \varphi}{\varphi_{k}+\varphi}
$$

or

$$
r=r_{0}+\frac{2\left(r_{1}-r_{0}\right)}{\frac{\varphi_{k}}{\varphi}+1}
$$

This equation, as can be seen, is the recording of a hyperbolic spiral. Its real fragment (considered herewith) is determined by the radius $r$, variable in the limits from $r_{0}$ to $r_{1}$; then there is the angle $\varphi$, with the variability in the limits from zero to $\varphi_{1}$.

Next the velocities: both angular $\omega$ and radial $v_{r}$ should be determined; they are of course the first derivatives corresponding with these directions of magnitudes, recorded by the formulae (11) and (21). Therefore

$$
\begin{gathered}
\omega=\frac{d \varphi}{d t}=\frac{\varphi_{k}}{T} e^{\frac{t}{T}}=\omega_{0} e^{\frac{t}{T}} \\
v_{r}=\frac{d r}{d t}=\frac{2\left(r_{1}-r_{0}\right)}{T} e^{-\frac{t}{T}}=v_{r}^{0} e^{-\frac{t}{T}}
\end{gathered}
$$

Furthermore, one should provide with the analytic record of accelerations: angular $\varepsilon$ and radial $p_{r}$. These magnitudes, in turn, are the first derivatives of the velocity, or the second derivatives of the path length. Therefore

$$
\begin{gathered}
\varepsilon=\frac{d^{2} \varphi}{d t^{2}}=\frac{d \omega}{d t}=\frac{\varphi_{k}}{T^{2}} e^{\frac{t}{T}}=\frac{\omega_{0}}{T} e^{\frac{t}{T}}=\varepsilon_{0} e^{\frac{t}{T}} \\
p_{r}=\frac{d^{2} r}{d t^{2}}=\frac{d v_{r}}{d t}=\frac{2\left(r_{1}-r_{0}\right)}{T^{2}} e^{-\frac{t}{T}}=\frac{v_{r}^{0}}{T} e^{-\frac{t}{T}}=p_{r}^{0} e^{-\frac{t}{T}}
\end{gathered}
$$

For the unstable state, if $t=t_{1}=T \ln 2$, the corresponding velocities and accelerations assume the following forms: 


$$
\begin{gathered}
\omega_{1}=2 \omega_{0} \\
v_{r}^{1}=\frac{v_{r}^{0}}{2} \\
\varepsilon_{1}=2 \varepsilon_{0} \\
p_{r}^{1}=\frac{p_{r}^{0}}{2}
\end{gathered}
$$

All these derivative kinetic magnitudes have been illustrated graphically (Fig. 4). One may notice, that for the angular motion, which is accelerated, they grow; the decreasing configuration relates to the radial motion, which is retarded. Of course, these changes are related to the body motion in the space-time. Later, in the uniform motion, they are invariable, constant.
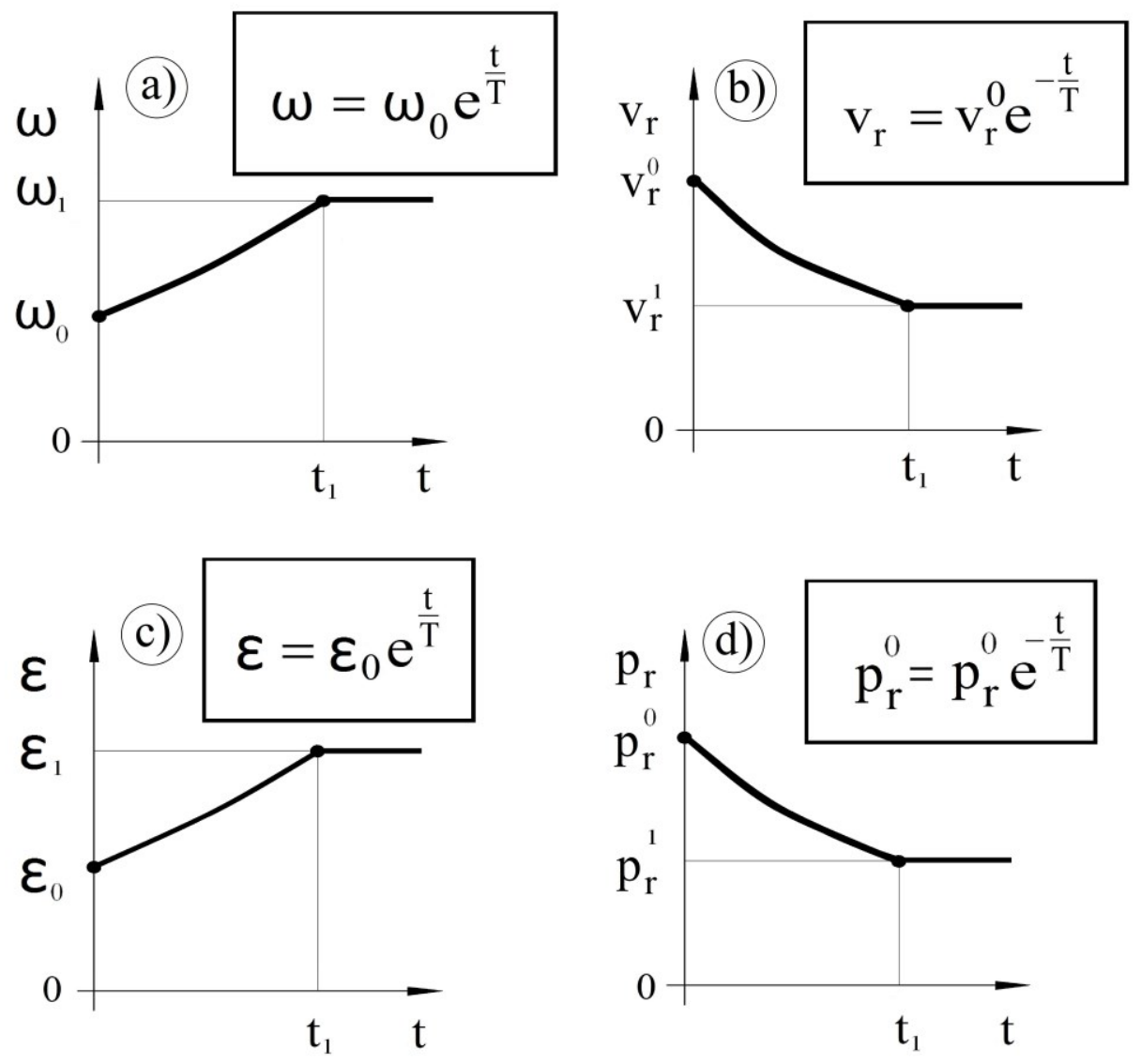

Fig. 4. Indicatrisse of dependences of angular velocity (a), radial velocity (b), angular acceleration (c), and radial acceleration $(\mathrm{d})$, on time 
The radial velocity $v_{r}$ at the same time is the relative velocity $v_{w}$, the radial acceleration $p_{r}$ is the relative acceleration $p_{w}$. In the peripheral direction the transportation velocity occurs which is connected with the angular velocity $\omega$ by the following dependence:

$$
v_{u}=\omega \cdot r
$$

Appearing in the same direction the transportation acceleration $p_{u}$ is comprised by the following dependence:

$$
p_{u}=\varepsilon \cdot r
$$

In the radial direction the known centrifugal acceleration $p_{o}$ occurs; it is expressed by the following formula:

$$
p_{o}=\omega^{2} \cdot r
$$

To close-up the matter, it is worthy presenting now the vector and scalar connections between the magnitudes occurring on the directions: radial and peripheral; in Fig. 5. the graphical presentation is given.

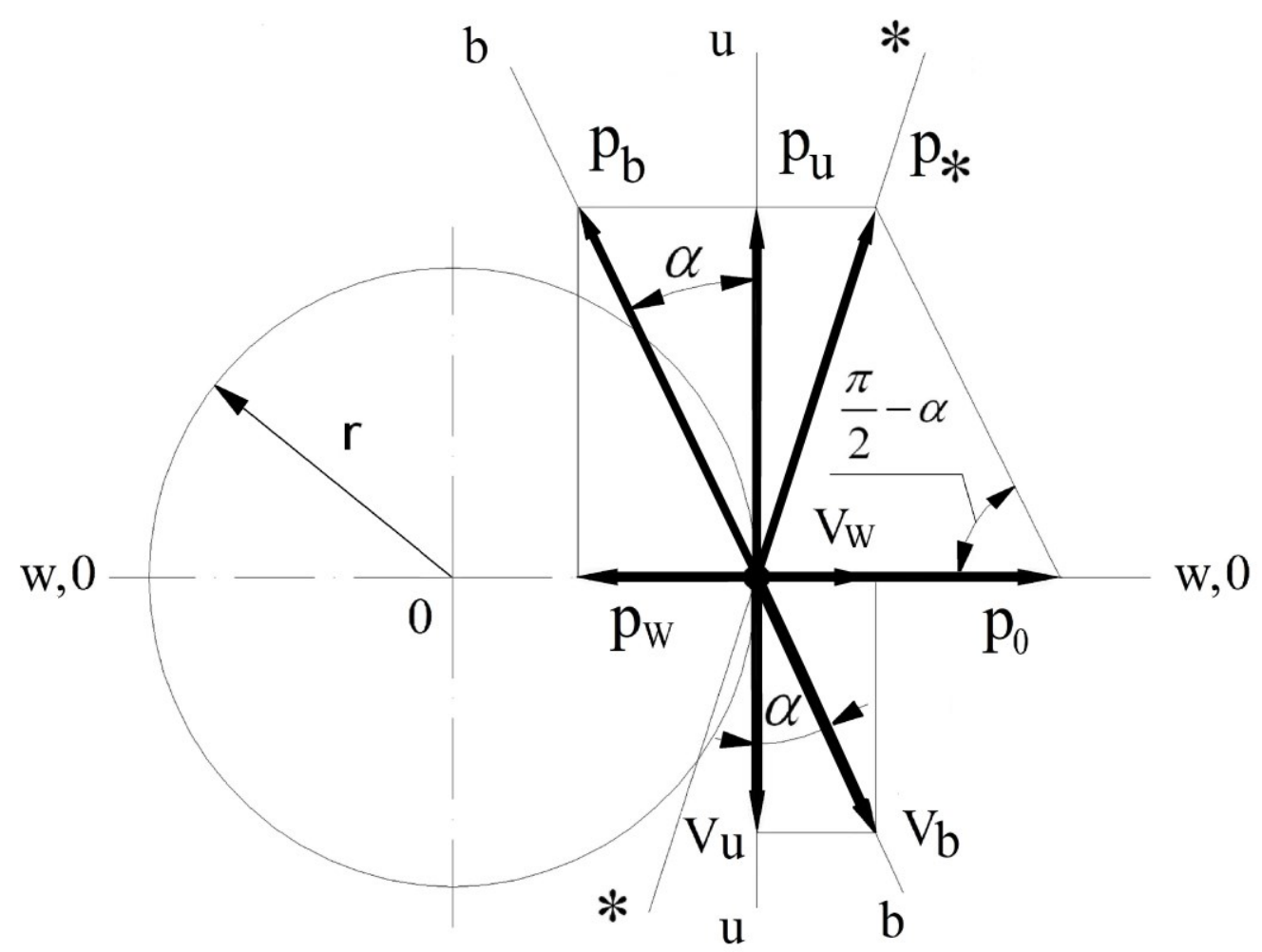

Fig. 5. Velocity and acceleration vectors of working element on the directions: relative $\mathrm{w}-\mathrm{w}$ and centrifugal $\mathrm{o}-\mathrm{o}$; absolute $\mathrm{b}-\mathrm{b}$; transportation $\mathrm{u}-\mathrm{u}$, and resultant $*-*$ 
The vector sum of velocities $\vec{v}_{u}$ and $\vec{v}_{w}$ gives the absolute velocity $\vec{v}_{b}$, so

$$
\vec{v}_{b}=\vec{v}_{w}+\vec{v}_{u}
$$

This kind of the accelerations sum on the mentioned directions gives the absolute acceleration, then

$$
\vec{p}_{b}=\vec{p}_{w}+\vec{p}_{u}
$$

The centrifugal acceleration $p_{o}$, should be added to the mentioned above ones, with the sum of all of them resulting in the total acceleration $p_{*}$. Therefore

$$
\vec{p}_{*}+\vec{p}_{w}+\vec{p}_{u}+\vec{p}_{o}
$$

or shorter, after taking into account (38)

$$
\vec{p}_{*}=\vec{p}_{b}+\vec{p}_{o}
$$

The scalar recording of these connections may be written as follows:

$$
\begin{gathered}
v_{b}=\sqrt{\left(v_{w}\right)^{2}+\left(v_{u}\right)^{2}} \\
p_{b}=\sqrt{\left(p_{w}\right)^{2}+\left(p_{u}\right)^{2}}
\end{gathered}
$$

The relation between the total acceleration $p_{*}$ and the accelerations: absolute $p_{b}$ and centrifugal $p_{o}$ results from the cosine theorem (Carnot), namely:

$$
\left(p_{*}\right)^{2}=\left(p_{o}\right)^{2}+\left(p_{b}\right)^{2}-2 p_{o} \cdot p_{b} \cos \left(\frac{\pi}{2}-\alpha\right)
$$

where the angle $\alpha$ is comprised between the velocities of: transformation $v_{u}$ and absolute $v_{b}$; as well as between these kind of accelerations.

It is worthy expressing the relationship between the transportation acceleration and the velocities: angular $\omega$ and relative $v_{w}$, for the energetic body states, i.e. on the radii $r_{0}$ and $r_{1}$. According to the formula (35) the accelerations for these radii equal as follows:

$$
p_{u}^{0}=\varepsilon_{0} \cdot r_{0}
$$




$$
p_{u}^{1}=\varepsilon_{1} \cdot r_{1}
$$

By regarding $\varepsilon_{0}=\omega_{0}: T$, that results from the formula (28); and then $\varepsilon_{1}=2 \varepsilon_{0}$, which is given by the formula (32); the dependences (44) and (45) may be presented as follows:

$$
\begin{aligned}
& p_{u}^{0}=\frac{r_{0}}{T} \omega_{0} \\
& p_{u}^{1}=\frac{2 r_{1}}{T} \omega_{0}
\end{aligned}
$$

Then one take advantage resulting from the formula (27), that is

$$
v_{r}^{0}=v_{w}^{0}=\frac{2\left(r_{1}-r_{0}\right)}{T}
$$

from which

$$
\frac{r_{0}}{T}=\frac{2 r_{1}}{T}-v_{w}^{0}
$$

and

$$
\frac{2 r_{1}}{T}=\frac{2 r_{0}}{T}+v_{w}^{0}
$$
finally

By substituting (49) and (50) to the formulae (46) and (47), respectively, one obtains

$$
p_{u}^{0}=\omega_{0}\left(\frac{2 r_{1}}{T}-v_{w}^{0}\right)
$$

and

$$
p_{u}^{1}=\omega_{0}\left(\frac{2 r_{0}}{T}+v_{w}^{0}\right)
$$




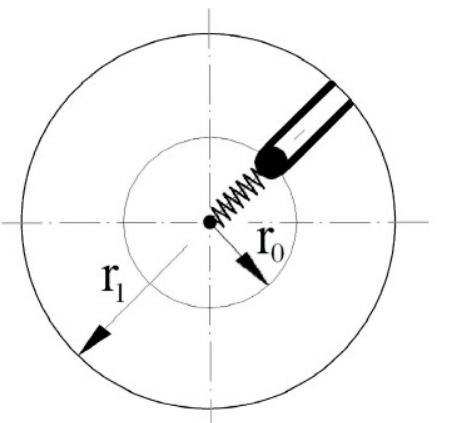

$$
\mathrm{p}_{\mathrm{u}}^{0}=\mathrm{w}_{0}\left(\frac{2 \mathrm{r}_{1}}{\mathrm{~T}}-\mathrm{v}_{\mathrm{w}}^{0}\right)
$$

$$
\mathrm{p}_{\mathrm{u}}^{\mathrm{l}}=\mathrm{w}_{0}\left(\frac{2 \mathrm{r}_{0}}{\mathrm{~T}}+\mathrm{v}_{\mathrm{w}}^{0}\right)
$$
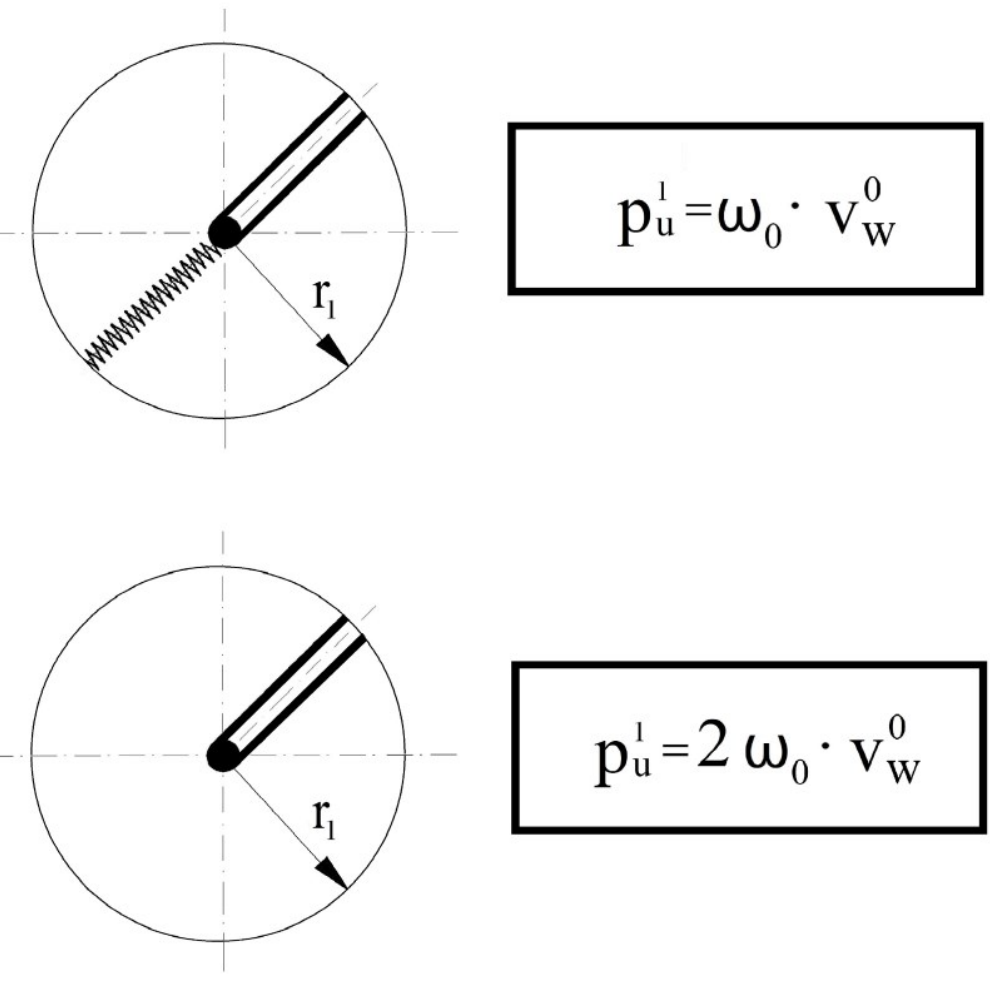

$$
\mathrm{p}_{\mathrm{u}}^{1}=2 \mathrm{w}_{0} \cdot \mathrm{v}_{\mathrm{w}}^{0}
$$

Fig. 6. Schemes of fixations of working element and corresponding descriptions of transportation acceleration

For a particular case, if $r_{0}=0$, according to the formula (48), one obtains

$$
v_{r}^{0}=\frac{2 r_{1}}{T}
$$

then

$$
\frac{2 r_{1}}{T}=v_{w}^{0}
$$


that results from the formula (50). Therefore, substituting to (47), one obtains

$$
p_{u}^{1}=\omega_{0} \cdot v_{w}^{0}
$$

In case if the working element is not retarded (by a spring) in the radial direction, then in both these directions (radial and angular) it will move with the accelerated motion. Thus

$$
\begin{gathered}
\varepsilon_{1}=2 \varepsilon_{0}=2 \frac{\omega_{0}}{T} \\
p_{u}^{1}=\varepsilon_{1} \cdot r_{1}=2 \omega_{0} \frac{r_{1}}{T}=2 \omega_{0} \cdot v_{w}^{0}
\end{gathered}
$$

The particular kinds of fixations of the working element differ substantially as to the stable working state (Fig. 6). It may be noticed, only for the first kind of the body fixation, there are two terminal transportation accelerations, related to the stable and unstable states of the body. For the remaining kinds of fixations and positioning of the considered element, where $r_{0}=0$, the system is characterized by only one terminal transportation acceleration (for the unstable state).

\section{CONCLUSION}

In the summary it is worthy noting an extremely essential meaning of the considered motion phenomenon of the flexibly fixed working element of the rotating tool. Broad and adequate description of such a complex motion of the determined elements of a material system possesses not only a cognitive value; the outcome of that reasoning may be carried out on the grounds of practical applications, by analyzing the behaviours of rotating elastic abrasive tools, as well as the design elements.

It should be stressed, that different variants of application of the working element and corresponding descriptions of the transportation acceleration have been considered. Thus it was possible to notice that particular situation with the description relating to the so called Coriolis acceleration. It appears that the acceleration is simply the transportation acceleration, though for a determined system.

All the presented solutions are the starting point to further analyses; they are the analyses covering the dynamics and kinematics of the considered material system, when the mass and all forces would be referred to.

\section{References}

[1] Eine ,weiche“ Schleifscheibe zum Polieren und Mikroentgraten, Prospectus of SLIP-NAXOS, 2002.

[2] Elastisch gebundene Schleifmittel, Catalogue ARTIFEX, 2003.

[3] Elastic polishing tools, Prospectus of Fabryka Tarcz Ściernych at Grodzisk Mazowiecki, 2001. 
[4] Programme Kronem-R-Flax Abrasifs, Prospectus of KLINGSPOR, 2004.

[5] Schleifen und Polieren, Catalogue of LUKAS, 2005.

[6] Systems of abrasive materials, Programme of delivery. Catalogue of 3M, 2000.

[7] H. Krug, G. Honcia, Werkstattstechnik 2 (1964) 53-59.

[8] K. Woźniak, Z. Pluta, Werkstatt und Betrieb 120(1) (1987) 55-58.

[9] Z. Pluta, Werkstatt und Betrieb 121(6) (1988) 487-489.

[10] Zdzisław Pluta, Fundamentals of surface smoothing by elastic abrasive wheels (in Polish). Edit. Office PolitechnikA Koszalińska, Koszalin, 2007.

[11] Zdzisław Pluta, Handle to mount flat grinding wheels of considerable susceptibility (in Polish). Patent RP No. 171852 (30.06.1997).

[12] Zdzisław Pluta, Fixation of elastic abrasive wheels of considerable susceptibility (in Polish). Proc. of 16th Sci. School of Abrasive Treatment. Koszalin, 1993, 67-74.

[13] Zdzisław Pluta, Head to mechanical smoothing treatment (in Polish). Patent PR No. 127473 (20.12.1984).

[14] W. Tryliński, Fine mechanisms and precision devices. Basics of design (in Polish), WNT, Warszawa, 1964 (2nd ed.).

[15] Z. Mrugalski (ed.), Design of devices and precision instruments (in Polish) Wydawnictwo Naukowo-Techniczne, Warszawa, 1996.

[16] R. Kurowski, M.E. Niezgodziński, Materials resistance (in Polish). PWN, Warszawa, 1968, 7th ed.

[17] Zdzisław Pluta, Tadeusz Hryniewicz, Intern. J. Adv. Manuf. Technol. 51 (2010) 217-223, DOI: 10.1007/s 00170-010-2595-5. 